\title{
The Influence of Vibration on Friction: A Contact-Mechanical Perspective
}

\author{
Mikhail Popov ${ }^{1,2 *}$ \\ ${ }^{1}$ Department of Continuum Mechanics and Constitutive Theory, Technische Universität Berlin, Berlin, Germany, ${ }^{2}$ Tomsk \\ State University, Tomsk, Russia
}

A unified model for active control of static and sliding friction by normal, tangential, and transverse oscillations is discussed, building on a series of past publications. The model in question is quasi-static, uses Amontons friction and takes into account contact stiffness in both normal and tangential directions. This makes the model fully macroscopic, which stands in contrast to Prandtl-Tomlinson-derived microscopic models that seem to be the currently preferred explanation for the influence of vibration on friction. While many technical details and numerical simulations based on our model have already appeared in a series of publications, here we attempt to give a high-level overview and discuss the main properties of friction under oscillation as generally as possible, while making a

OPEN ACCESS

Edited by:

Marco Paggi,

IMT School for Advanced Studies

Lucca, Italy

Reviewed by:

Shingo Ozaki,

Yokohama National University, Japan

Varvara Romanova,

Institute of Strength Physics and Materials Science (ISPMS SB RAS),

Russia

Vladislav Aleshin,

UMR8520 Institut d'électronique, de

Microélectronique et de

Nanotechnologie (IEMN), France

*Correspondence:

Mikhail Popov

mpopov@fastmail.fm

Specialty section:

This article was submitted to

Tribology,

a section of the journal

Frontiers in Mechanical Engineering

Received: 29 May 2020

Accepted: 14 July 2020

Published: 20 August 2020

Citation:

Popov M (2020) The Influence of Vibration on Friction: A Contact-Mechanical Perspective.

Front. Mech. Eng. 6:69.

doi: 10.3389/fmech.2020.00069 minimum of assumptions.

Keywords: vibration, friction, contact mechanics, active control, stick-slip actuation

\section{INTRODUCTION}

The fact that vibration can be used to significantly reduce the force of friction has been known since at least the 1950s (Fridman and Levesque, 1959). Since then, the effect has been studied extensively and exploited in many practical applications. Classical examples are to be found in wire drawing (Murakawa and Jin, 2001; Siegert and Ulmer, 2001), press forming (Eaves et al., 1975; Ashida and Aoyama, 2007), cutting (Thoe et al., 1998; Eggers et al., 2004), and other machining processes. Also well-known is the use of vibration for stabilization of system dynamics, e.g., suppression of brake squeal (Müller and Ostermeyer, 2007) and cornering noise (Heckl and Huang, 2000).

There are also a number of advanced applications that move beyond simple reduction of sliding or static friction, and involve vibration-driven directed transport or exact positioning (Popov, 2017). The most famous example of this are traveling wave motors (Schmidt et al., 1996; Storck et al., 2002), which are used to adjust focus in camera lenses, among many other applications. Similar principles are employed in high-precision linear actuators and positioning systems (Socoliuc et al., 2006), vibrational conveyors (Gaberson, 1971, 1972), and other types stick-slip drives.

The above examples are only a small sample of technical applications at the intersection of friction and vibration. Correspondingly, there is a large body of existing research in this field (see e.g., Pohlman and Lehfeldt, 1966; Godfrey, 1967; Storck et al., 2002; Chowdhury and Helali, 2008). Most of it is practical in nature, even though several well-known theoretical models have been proposed as well (De Wit et al., 1995). However, it is the contention of the author that an important factor is missing from currently popular models: the compliance of the contact and its interaction with the applied oscillation. The currently prevailing tendency is to ascribe the reduction of friction by vibration mostly to processes at the micro-scale (Popov et al., 2010). However, here we will argue that the primary (but not necessarily exclusive) mechanism is to be found on the macroscale, in ordinary contact mechanics. It should be noted that this does not automatically invalidate previous work. In fact, it seems likely that a truly accurate model will be multiscale, combining both macroscopic dynamics and microscopic processes. 


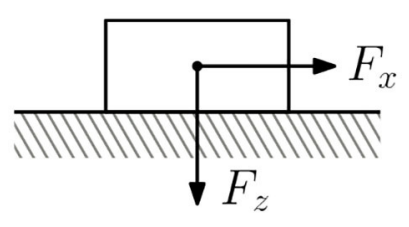

FIGURE 1 | Static friction model. The intrinsic coefficient of friction in the contact is $\mu_{0}$. The $y$-axis points out of the plane.

The primary advantage of our model is its simplicity. It relies only on macroscopic contact mechanics and introduces no new physics. In fact, it is likely to be the simplest possible model that is rich enough to describe almost the full range of behaviors exhibited by friction under the influence of external vibration. For this reason, the present paper can be seen as an exercise in minimalism, attempting to cover as much phenomenological ground as possible with a minimum of assumptions and variables.

\subsection{Contributions}

This work draws heavily on results recently published in a series of papers with participation of the present author (Mao et al., 2017; Popov et al., 2017; Benad et al., 2018a,b; Popov and Li, 2018). While there is substantial overlap with these papers, the present work is organized differently, seeking to present a "big picture" view without getting bogged down in details. Several results have been generalized from previous publications, while the discussion of the influence of oscillation waveforms has, to the best knowledge of the author, not previously appeared in the literature.

\section{STATIC FRICTION}

To warm up, we consider static friction. This case is much simpler than the sliding case and leads to some satisfyingly general results. The system under consideration consists of a body resting on a plane (Figure 1). The body is pressed into the plane with a force $F_{z}$ and pulled sideways with a force $F_{x}$. The coefficient of friction between the body and the plane is assumed to be constant and equal to $\mu_{0}$. The body remains at rest while

$$
\left|F_{x}\right|<\mu_{0} F_{z}
$$

where $\mu_{0} F_{z}$ is the critical force at which the body just begins to slide. The static coefficient of friction is defined as the ratio of this critical force to the normal load. In the absence of oscillation, it is equal to $\mu_{0}$ :

$$
\mu_{s}=\mu_{0}
$$

Things get slightly more interesting when we add an oscillatory force component. If the force oscillation acts normal to the plane, we denote it by $A_{z} g(t)$, where $A_{z}$ is the amplitude. The stick condition in that case needs to be amended to:

$$
\left|F_{x}\right|<\mu_{0}\left(F_{z}+A_{z} g(t)\right)^{+}
$$

The (..) $)^{+}$notation denotes the ramp function, which clips negative values to zero. It is necessary because the normal force does not turn negative when contact is lost.

It is easy to see that the critical force is reduced relative to the non-oscillatory case, since the above inequality must hold at all times, including the times when the normal force drops below its mean value $F_{z}$. In other words, static friction is limited by the minimum of normal force encountered during the oscillation. For the coefficient of static friction under normal oscillation we thus obtain:

$$
\mu_{s, z}=\mu_{0}\left(1-A_{z} / F_{z}\right)^{+}
$$

In a similar fashion, we can add an oscillatory component $A_{x} g(t)$ that is aligned with the tangential force $F_{x}$. This results in the stick condition

$$
\left|F_{x}+A_{x} g(t)\right|<\mu_{0} F_{z}
$$

Note that this inequality is only satisfiable when $A_{x}<\mu_{0} F_{z}$. Otherwise the body starts to slide in place and the contact loses its ability to statically sustain a lateral force. Thus, $\mu_{s}$ can be expressed as:

$$
\mu_{s, x}=\left(\mu_{0}-A_{x} / F_{z}\right)^{+}
$$

Notice the slight difference between this result and Equation (4). In particular, note that a tangential oscillation will reduce $\mu_{s}$ by a larger amount than a normal oscillation of the same amplitude if $\mu_{0}<1$, and by a smaller amount otherwise.

Transverse oscillations are also able to reduce static friction. This case is qualitatively similar to that of tangential oscillation, with the difference that we need to use the vector norm of the in-plane forces instead of adding them directly:

$$
F_{x}^{2}+\left(A_{y} g(t)\right)^{2}<\left(\mu_{0} F_{z}\right)^{2}
$$

Once again, stick is impossible if $A_{y} \geq \mu_{0} F_{z}$, and for the static coefficient of friction we obtain:

$$
\mu_{s, y}=\sqrt{\left(\mu_{0}^{2}-A_{y}^{2} / F_{z}^{2}\right)^{+}}
$$

One particularly useful thing about these results is that they are quite general, and in particular independent of contact geometry, frequency of oscillation, and the shape of the oscillation waveform.

\subsection{Static Friction Under Superimposed Oscillation}

Things become considerably less transparent when we consider simultaneous oscillation in multiple directions. The stick condition itself does not change much, and in the most general case can be expressed as:

$$
\left(F_{x}+A_{x} g_{x}(t)\right)^{2}+\left(A_{y} g_{y}(t)\right)^{2}<\mu_{0}^{2}\left(F_{z}+A_{z} g_{z}(t)\right)^{2}
$$

Unfortunately, actually finding the maximal $F_{x}$ that still satisfies this inequality at all times quickly becomes unwieldy, leading to 


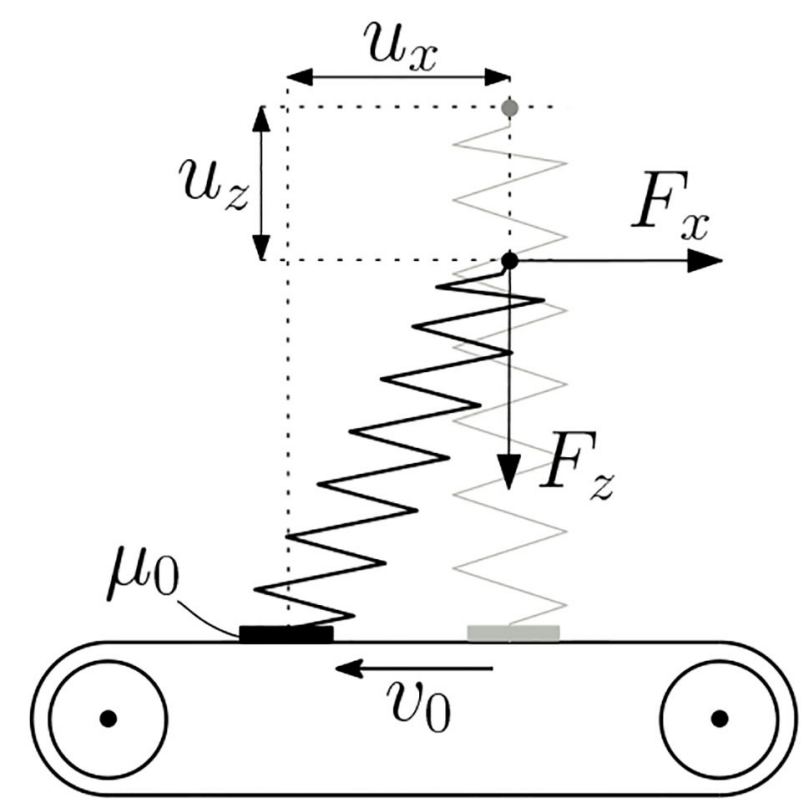

FIGURE 2 | A single massless spring, which serves as a minimal model of a sliding frictional contact. The sliding velocity is constant, while the vertical coordinate oscillates. Amontons friction with the constant coefficient of friction $\mu_{0}$ is assumed in the contact point.

a large number of case distinctions-if a closed-form solution is possible at all. In addition, when the compliance of the contact is taken into account, the static coefficient of friction may become negative, in the sense that a constant force needs to be applied to prevent the contact from sliding. This effect is what frictional drives and actuators are based on. For an analysis of this case the reader is referred to Popov and Li (2018). In this paper, however, we ignore superimposed oscillation.

\section{SLIDING FRICTION UNDER NORMAL OSCILLATION}

The key feature of the model that we use to describe dynamic friction is that the compliance of the contact is taken into account. In the initial formulation, the contact is modeled as a single Hookean spring that has an associated normal stiffness $k_{z}$ and a lateral stiffness $k_{x}$ (Figure 2). This is a reasonable approximation of a flat-ended cylinder in contact with a plane. The model can also be extended to cover arbitrary curved contacts with the help of the Method of Dimensionality Reduction (Popov and Heß, 2016). However, for a general analysis, a single spring is quite sufficient.

The model considered here is displacement-controlled and quasi-static. A force-controlled and/or inertial model can be formulated within the same framework, which, however, leads to certain complications (e.g., resonances) that are outside the focus of the present paper. For an analysis of such a model, the reader is referred to Mao et al. (2017).
The kinematics of the model is as follows: The contact spring is pulled over a flat plane with a constant velocity $v_{0}$, although for convenience we consider the spring to be stationary, while the substrate slides underneath it. The normal displacement $u_{z}$ of the spring is measured relative to the state of unstressed first contact with the substrate. $u_{z}(t)$ represents the externally applied oscillation and is thus given explicitly. The lateral displacement $u_{x}$, on the other hand, depends on the current state of the system and is the only unknown variable.

We assume that Amontons' law of friction (with a constant coefficient of friction $\mu_{0}$ that is the same for both static and sliding friction) holds in the contact point. In general, this may be an unrealistic assumption. However, the use of a constant coefficient of friction not only simplifies calculations, but also eliminates all possible micro-scale influences from the model. Since one of the primary aims of this paper is to advertise the feasibility of a purely macroscopic theory of friction under oscillation, making $\mu_{0}$ constant is actually a prerequisite.

The effective coefficient of friction $\bar{\mu}$, which is to be determined in the sequel, is defined as the average tangential force exerted by the spring divided by the average normal force:

$$
\bar{\mu}=\frac{\left\langle F_{x}(t)\right\rangle}{\left\langle F_{z}(t)\right\rangle}
$$

where $\langle.$.$\rangle denotes averaging over one period of oscillation.$

Previous publications on the topic assumed that the imposed normal oscillation is harmonic, so as to simplify analysis. However, this turned out to be an unnecessary restriction, so here we will work with a general periodic function that is parameterized as follows:

$$
u_{z}(t)=\bar{u}_{z}+A_{z} w(f t)
$$

Here $\bar{u}_{z}$ is the mean indentation, $A_{z}$ is the amplitude and $f$ the frequency of the oscillation. $w(\varphi)$ is a dimensionless function describing the "shape" of the oscillation, with $\varphi=f t$. The waveform $w$ is normalized such that it is zero-mean, with a period of 1 and a minimum value of -1 . Note however, that the maximum of $w$ is left unconstrained.

\subsection{Pure Sliding}

While the behavior of a frictional couple under oscillation has its complexities in general, there are two extreme cases that lend themselves to easy and precise analysis: One of them, static friction, was already discussed above. The second, pure sliding, is briefly discussed here. The most important thing about pure sliding is that oscillations do not influence the coefficient of friction in that mode. This can be easily seen from the fact that the instantaneous tangential force is uniquely defined during slip $\left(F_{x}=\mu_{0} F_{z}\right)$, from which the effective coefficient of friction is immediately obtained:

$$
\bar{\mu}_{\text {slip }}=\frac{\left\langle F_{x}(t)\right\rangle}{\left\langle F_{z}(t)\right\rangle}=\frac{\left\langle\mu_{0} F_{z}(t)\right\rangle}{\left\langle F_{z}(t)\right\rangle}=\mu_{0}
$$

Irrespective of how complex the dependence $F_{z}(t)$ may be, it always cancels out-by linearity of sliding friction. While 
this result may seem unimpressive by itself, it establishes an important "boundary condition" for the more general case of friction with stick-slip. Also, as in the static case, the coefficient of friction in pure slip has the important property of not being dependent on contact geometry and oscillation parameters. The result $\bar{\mu}_{\text {slip }}=\mu_{0}$ is also valid for tangential and combined normal/tangential oscillations. It can also be shown to be valid in the inertial case (Mao et al., 2017). However, transverse oscillations do not, strictly speaking, have this limiting case, although the deviation becomes negligible at high velocities. This will be discussed in more detail later.

\subsection{Stick-Slip}

Two extreme points have now been established: pure stick (static friction) and pure slip (plateau). Reason suggests that there is also something in between. It would be physically implausible for the coefficient of friction under oscillation to "snap" from near zero back to $\mu_{0}$ due to arbitrarily slow sliding. And this is in fact not observed experimentally: At a given amplitude and frequency, the static coefficient of friction is lowest, and then smoothly increases with the sliding velocity until reaching a plateau of sorts. Fortunately, the transition region can also be described in our model. Unsurprisingly, it is dominated by stick-slip.

Let us now consider this phenomenon in more detail. During sliding, we have $F_{x}=\mu_{0} F_{z}$, which can also be written as $k_{x} u_{x}(t)=\mu_{0} k_{z} u_{z}(t)$. Substituting $u_{z}$ from Equation (11) and rearranging gives us the lateral displacement and velocity of the contact point:

$$
\begin{aligned}
& u_{x}(t)=\mu_{0} \frac{k_{z}}{k_{x}}\left(\bar{u}_{z}+A_{z} w(f t)\right) \\
& \dot{u}_{x}(t)=\mu_{0} \frac{k_{z}}{k_{x}} A_{z} f w^{\prime}(f t)
\end{aligned}
$$

A transition from slip to stick happens when the relative motion between the substrate and the contact point vanishes, i.e., when $\dot{u}_{x}(t)=v_{0}$. From this condition, the point of stick onset can be determined:

$$
\varphi_{1}=f t_{1}=\left(w^{\prime}\right)^{-1}\left(\frac{k_{x} v_{0}}{\mu_{0} k_{z} A_{z} f}\right)
$$

It becomes obvious that $\varphi_{1}$ is a function of a single compound variable, which combines all parameters of the system, except $\bar{u}_{z}$. To simplify further calculations, we introduce some dimensionless variables, $\alpha$ (corresponding to amplitude), $\beta$ (corresponding to velocity), and $\varphi$ (phase):

$$
\begin{aligned}
\alpha & =\frac{A_{z}}{\bar{u}_{z}} \\
\beta & =\frac{k_{x} v_{0}}{\mu_{0} k_{z} A_{z} f} \\
\varphi & =f t
\end{aligned}
$$

Using these variables, the static coefficient of friction (Equation 4) can be expressed as $\mu_{s, x}=\mu_{0}(1-\alpha)^{+}$, while Equation (15) can be written as

$$
\varphi_{1}=\left(w^{\prime}\right)^{-1}(\beta)
$$

Noting that $\beta$ is a positive quantity and assuming that $w$ is differentiable (but not necessarily invertible-there can be multiple stick events), it can be seen that the above equation has solutions if

$$
\beta<\max _{\varphi} w^{\prime}(\varphi)=\beta_{c}
$$

where $\beta_{c}$ denotes the critical value that separates the stickslip region from the continuous sliding region. A harmonic oscillation, for example, has $\beta_{c}=2 \pi$, while a right-leaning sawtooth function has $\beta_{c}=2$, which is in fact the smallest possible value. The larger $\beta_{c}$, the more effective the waveform is at reducing friction at high velocities, but more on that later.

Once stick is initiated, the contact point is dragged along by the substrate with velocity $v_{0}$, so that the tangential displacement and force increase linearly with time:

$$
F_{\text {stick }}(t)=\mu_{0} F_{z}\left(t_{1}\right)+k_{x} v_{0}\left(t-t_{1}\right)
$$

This continues while the condition for static friction holds:

$$
F_{\text {stick }}(t)<\mu_{0} F_{z}(t)
$$

Trivial as it is, this inequality lies at the core of reduction of friction in our model. It serves as the sole source of nonlinearity that allows the system to break free of the trivial solution exemplified by Equation (12). With pure slip, the spring force is always equal to $\mu_{0} F_{z}(t)$, while in stick-slip it is sometimes lower, which leads to lower average force and coefficient of friction (see also Figure 3). Another way of looking at it is that the contact point stands still when the normal force is highest, and covers more distance when the normal load diminishes. This leads to lower energy dissipation over the same distance. The whole process is somewhat similar to walking, where one leg carries the load without dissipation, while the other is lifted and advanced to the next position. Something analogous happens in our model, only there is just one "leg" and it is not necessarily lifted all the way.

The stick phase ends at time $t_{2}$ when the condition $F_{\text {stick }}\left(t_{2}\right)=$ $\mu_{0} F_{z}\left(t_{2}\right)$ is met. Expanding this condition yields

$$
\mu_{0} k_{z} u_{z}\left(t_{1}\right)+k_{x} v_{0}\left(t_{2}-t_{1}\right)=\mu_{0} k_{z} u_{z}\left(t_{2}\right)
$$

or, more conveniently,

$$
\frac{v_{0} k_{x}}{\mu_{0} k_{z}}\left(t_{2}-t_{1}\right)=u_{z}\left(t_{2}\right)-u_{z}\left(t_{1}\right)
$$

Substituting $u_{z}$ and $t=\varphi / f$, this can be rewritten as:

$$
\beta\left(\varphi_{2}-\varphi_{1}\right)=w\left(\varphi_{2}\right)-w\left(\varphi_{1}\right)
$$

Once again $\bar{u}_{z}$ cancels out, leaving us with a function of only $\beta$. Unfortunately, the equation is implicit and cannot be solved symbolically for $\varphi_{2}$ except in the simplest cases (sawtooth, square wave, etc). In the case of a harmonic oscillation, for example, Equation (25) takes the form $(\cos x=a+b x)$, which does not have a closed-form solution in terms of standard functions. Numerical solution is required in most cases. 


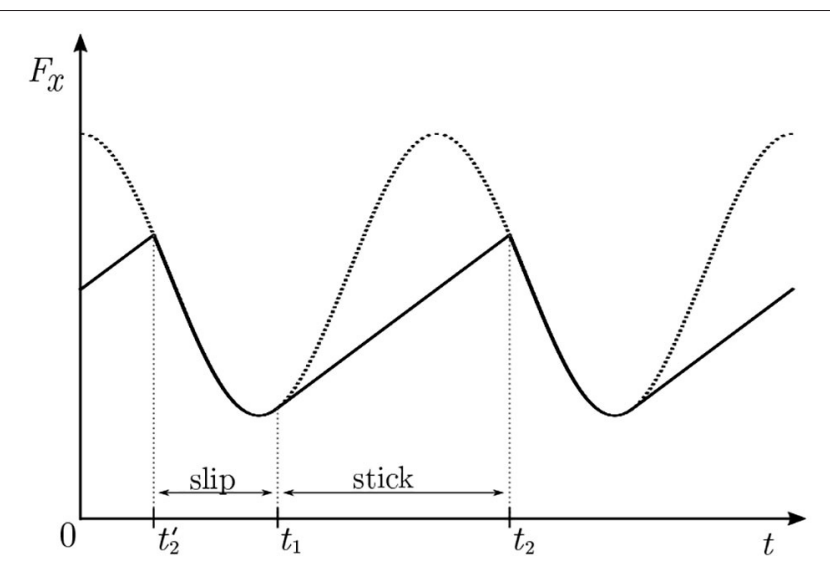

FIGURE 3 | Stick and slip under the influence of a harmonic oscillation. The dotted line represents the tangential force as it would be in pure slip $\left[F_{\text {slip }}=\mu_{0} F_{Z}(t)\right]$. The solid line is the actual tangential force in the presence of stick-slip. The stick phases are the straight segments, e.g., between $t_{1}$ and $t_{2}$, while slip phases are the sinusoidal segments, e.g., between $t_{2}^{\prime}$ and $t_{1}$, repeating periodically. Note that $F_{x} \leq F_{\text {slip }}$ everywhere, which is the origin of friction reduction in our model.

\subsection{Effective Coefficient of Friction}

We define the "macroscopic" or "effective" force of friction simply as the tangential force averaged over one period $T=1 / f$ :

$$
\left\langle F_{x}\right\rangle=\frac{1}{T} \int_{0}^{T} F_{x}(t) \mathrm{d} t
$$

However, it will become clear in a moment that it is more convenient to consider the difference or reduction of the force of friction relative to the state of continuous sliding:

$$
\Delta F_{x}=\left\langle F_{\text {slip }}\right\rangle-\left\langle F_{x}\right\rangle=\frac{1}{T} \int_{0}^{T}\left(F_{\text {slip }}(t)-F_{x}(t)\right) \mathrm{d} t
$$

Since $F_{x}$ only differs from $F_{\text {slip }}$ during the stick phase, we can tighten the integration bounds:

$$
\Delta F_{x}=\frac{1}{T} \int_{t_{1}}^{t_{2}}\left(\mu_{0} F_{z}(t)-F_{\text {stick }}(t)\right) \mathrm{d} t
$$

This form is convenient for numerical solution. However, some additional properties can gleaned by expanding $F_{\text {stick }}$ and $u_{z}(t)$ and making the substitution $\mathrm{d} t=T \mathrm{~d} \varphi$ :

$$
\begin{aligned}
\Delta F_{x} & =\frac{1}{T} \int_{t_{1}}^{t_{2}}\left(\mu_{0} F_{z}(t)-\mu_{0} F_{z}\left(t_{1}\right)-k_{x} v_{0}\left(t-t_{1}\right)\right) \mathrm{d} t \\
& =\frac{1}{T} \int_{t_{1}}^{t_{2}} \mu_{0} k_{z}\left(\bar{u}_{z}+A_{z} w(f t)-\bar{u}_{z}-A_{z} w\left(f t_{1}\right)\right. \\
& \left.-\frac{k_{x} v_{0}}{\mu_{0} k_{z}}\left(t-t_{1}\right)\right) \mathrm{d} t \\
& =\mu_{0} k_{z} A_{z} \int_{\varphi_{1}}^{\varphi_{2}}\left(w(\varphi)-w\left(\varphi_{1}\right)-\beta\left(\varphi-\varphi_{1}\right)\right) \mathrm{d} \varphi
\end{aligned}
$$

It becomes apparent that the expression for $\Delta F_{x}$ can be split into the dimensional factor $\mu_{0} k_{z} A_{z}$ and a dimensionless function $\Psi_{w}$ of a single variable:

$$
\Delta F_{x}=\mu_{0} k_{z} A_{z} \Psi_{w}(\beta)
$$

where

$$
\Psi_{w}(\beta)=\int_{\varphi_{1}}^{\varphi_{2}}\left(w(\varphi)-w\left(\varphi_{1}\right)-\beta\left(\varphi-\varphi_{1}\right)\right) \mathrm{d} \varphi
$$

We refrain from integrating this expression, since a closed-form solution is precluded by the lack of an explicit formula for $\varphi_{2}$. We merely draw attention to the fact that $\Delta F_{x}$ is invariant with respect to mean indentation. The same is not true for the coefficient of friction:

$$
\bar{\mu}=\frac{\left\langle\mu_{0} F_{z}\right\rangle-\Delta F_{x}}{\left\langle F_{z}\right\rangle}=\mu_{0}-\frac{\Delta F_{x}}{k_{z} \bar{u}_{z}}
$$

However, the dependence on $\bar{u}_{z}$ is incidental, merely reflecting the fact that $\Delta F_{x}$ is subtracted from different baselines of friction force. Using our dimensionless variables, the above can also be written in the following compact form:

$$
\bar{\mu}=\mu_{0}\left(1-\alpha \Psi_{w}(\beta)\right)
$$

Further, it can be shown that $\Psi_{w}$ is a fairly well-behaved function that has unit range and is monotonously decreasing and convex for all waveforms and any number of stick events per cycle of oscillation. However, space considerations prevent us from including a formal proof of these properties.

\subsection{Oscillation Waveforms}

The functional dependence (33) presented in the previous section permits an interesting observation: the overall strength of the friction reduction effect is primarily governed by the amplitude of the oscillation and not by the frequency. In principle, the effective coefficient of friction can be reduced to very low values, but that requires a force amplitude that is comparable to the mean normal force. Thus, the technique is not very useful for reducing friction in highly loaded contacts, e.g., rail-car or truck wheels.

Furthermore, a higher frequency cannot be used to compensate for small amplitude. However, frequency is still an important parameter, since it determines the "velocityresistance" of the effect: As has been pointed out before, the largest reduction is always seen in the static case, and becomes lower with increasing sliding velocity. The frequency determines the scaling of this decline, and a strong reduction can be achieved even at high sliding velocities if the frequency of the applied oscillation is high enough. However, frequency is not the only factor that determines this "velocity-resistance." The waveform of the oscillation is also quite important, which is why we briefly discuss it here.

By far the most important property of a waveform $w$ is the maximal positive value of its first derivative, or $\beta_{c}$. A rightleaning sawtooth function, for example, has $\beta_{c}=2$; a harmonic 
oscillation has $\beta_{c}=2 \pi$, which is slightly better; however, a leftleaning sawtooth function has $\beta_{c}=\infty$, which is ideal. An infinite value of $\beta_{c}$ implies that the oscillation will provide some measure of friction reduction at arbitrarily high velocities. While this is not really possible in practice, the general rule for waveform selection is nevertheless that the load should increase as fast as possible and then relax slowly. Thus, approximations of leftleaning sawtooth or the square wave are preferable to smooth and symmetric functions like the harmonic oscillation. Naturally, this recommendation is subordinate to practical technological constraints. For example, a high-amplitude harmonic oscillation could be generated by exciting a natural vibrational mode of the system, while a square wave would likely require more sophisticated equipment.

We conclude this section by giving $\Psi_{w}$ for a few common waveforms explicitly. For both sawtooth variants and the square wave $\Psi_{w}$ can be calculated in closed form. However, for most oscillations, including sinusoidal ones, this is not possible. Nonetheless, the function can easily be computed numerically for arbitrary waveforms, and so we include two empirical approximations for the harmonic oscillation, which were first obtained in Popov et al. (2017). The first approximation is slightly more accurate.

$$
\begin{aligned}
\Psi_{\mathrm{str}}(\beta) & =1-\frac{\beta}{2} \\
\Psi_{\mathrm{stl}}(\beta) & =\frac{2}{2+\beta} \\
\Psi_{\mathrm{sqr}}(\beta) & = \begin{cases}1-\beta / 8, & \text { for } \beta<4 \\
2 / \beta, & \text { for } \beta>4\end{cases} \\
\Psi_{\mathrm{sin}}(\beta) & \approx \frac{3}{4}\left(1-\beta / \beta_{c}\right)^{2}+\frac{1}{4}\left(1-\beta / \beta_{c}\right)^{4} \\
& \approx\left(1-\beta / \beta_{c}\right)^{2.4}
\end{aligned}
$$

For a visual comparison, the dependence of the coefficient of friction on $\beta$ is plotted in Figure 4 for all four of the above waveforms. To keep things simple, only the case of maximal friction reduction is shown $\alpha=1$, in which case Equation (33) reduces to $\bar{\mu}=\mu_{0}\left[1-\Psi_{w}(\beta)\right]$. This is why all curves show zero static friction. For other values of $\alpha$ the shapes of the curves would remain the same, but they would start at nonzero values of $\mu_{s}$ and would be scaled accordingly.

As a final remark, we note that there is a unique optimal waveform with regards to reduction of friction. It is given by the periodic extension of $\delta(\varphi)-1$, where $\delta$ is the Dirac delta function. This "impulse wave" is -1 everywhere, except for very short positive spikes (impulses) that occur with a period of 1 and each integrate to 1 , so that the average of the function is zero. With this degenerate waveform, the system slides most of the time, with only an infinitesimal stick phase at each spike, which implies that $\Psi$ is very close to 1 for all $\beta$ :

$$
\Psi_{\text {imp }}(\beta) \rightarrow 1
$$

Thus, we conclude that friction can be reduced, in principle, to an arbitrary degree even at high sliding velocities, by effectively

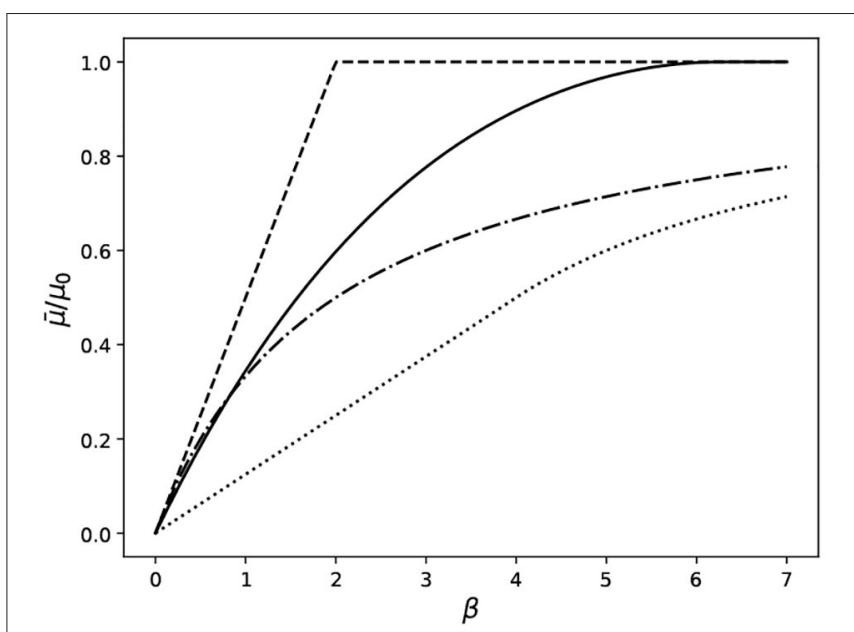

FIGURE 4 | Coefficient of friction under normal vibration with different waveforms, computed using Equation (33) and the individual influence factors Equations (34)-(37). Note that for all curves $\alpha=1$, which corresponds to maximal friction reduction. Legend: dashed line-right-leaning sawtooth function (Equation 34); solid line-harmonic oscillation (Equation 37); dash-dotted line-left-leaning sawtooth function (Equation 35); dotted line-square wave (Equation 36).

hopping over the surface. In practice, this approach will be limited by plastic deformation, radiation of elastic waves and the sheer difficulty of generating such an oscillation.

\section{TANGENTIAL AND TRANSVERSE OSCILLATIONS}

Most of this paper was devoted to reduction of friction by normal oscillations. This focus is explained partly by the fact that the normal case is easiest to analyze, and partly because normal oscillations are generally the most efficient way to reduce friction, out of the three possible directions. Nevertheless, both tangential (in the direction of sliding) and transverse (in-plane, but orthogonal to sliding) vibration can reduce friction. For detailed analysis of the tangential case the reader is referred to Popov and Li (2018) and for the transverse case to Benad et al. (2018a). Here we only present some highlights and point out the major differences between normal oscillations and the other two modes.

In the tangential oscillation case the normal indentation is kept constant while an oscillatory component is added to the base of the spring. Sliding friction under such conditions can proceed in three modes: (I) pure sliding, in which the effective coefficient of friction is equal to $\mu_{0}$, as argued previously. (II) simple stick-slip, which occurs for obvious reasons when the velocity amplitude is greater than the mean sliding velocity $\left(A_{x} f w^{\prime}(f t)>v_{0}\right)$. (III) multiple stick-slip, which occurs when the velocity amplitude is much larger than $v_{0}$, so that the contact point slides back-and-forth in each cycle, going through two stick and slip phases each. 
The most important difference between friction reduction by normal and tangential oscillations is that normal oscillations actually reduce the total dissipated energy through a walking-like mechanism, while tangential oscillations do not. The author is not aware of a good analogy to visualize the mechanism in the tangential case. But it is clear that, since the normal load (and therefore the force of sliding friction) is constant, the dissipated energy is simply friction force times distance (in mode II). Although the effective coefficient of friction (i.e., average spring force) may be lowered, the missing energy must be supplied by the oscillator. In mode III, when the amplitude is large enough to cause in-place sliding, the total sliding distance actually increases, and the total energy expenditure becomes larger than without oscillations, even though the effective coefficient of friction will still appear lower than $\mu_{0}$.

Friction reduction by transverse oscillation always operates in something like mode III of tangential oscillation: it causes additional sliding in the direction orthogonal to the main sliding motion, thereby increasing the total path and therefore energy expenditure. However, the apparent coefficient of friction is reduced, because the magnitude of the local friction force is still limited to $\mu_{0} F_{z}$, but now shared between the force components parallel and orthogonal to the main sliding direction. Thus, transverse oscillations are effectively "stealing" the friction vector from the slider, but at considerable expense of energy by the oscillator. This also accounts for the fact, mentioned previously, that the system never formally reaches the "invariant plateau" $\left(\bar{\mu}_{\text {slip }}=\mu_{0}\right)$ even at high sliding velocities, because the projection of the local friction force onto the sliding direction is always less than its total magnitude, so long as the transverse amplitude is non-zero. However, for sufficiently large sliding velocities this difference becomes very small, so for all practical purposes the plateau exists in the transverse case as well.

To summarize, normal oscillations are most effective at reducing dynamic friction and should be used in preference to the other directions. Not only do they actually reduce the total dissipated energy, but normal oscillations also act at right angles (by definition) to the sliding motion. Thus, they technically do not require energy to keep going. Of course, this is never quite the case in practice, but by exciting a resonant frequency the power needed to drive the oscillator can usually

\section{REFERENCES}

Ashida, Y., and Aoyama, H. (2007). Press forming using ultrasonic vibration. J. Mater. Process. Technol. 187, 118-122. doi: 10.1016/j.jmatprotec.2006.11.174

Benad, J., Nakano, K., Popov, V. L., and Popov, M. (2018a). Active control of friction by transverse oscillations. Friction 7, 1-12. doi: 10.1007/s40544-018-0202-1

Benad, J., Popov, M., Nakano, K., and Popov, V. L. (2018b). Stiff and soft active control of friction by vibrations and their energy efficiency. Forsch. Ingenieurw. 82, 331-339. doi: 10.1007/s10010-018-0281-1

Chowdhury, M. A., and Helali, M. (2008). The effect of amplitude of vibration on the coefficient of friction for different materials. Tribol. Int. 41, 307-314. doi: 10.1016/j.triboint.2007.08.005

De Wit, C. C., Olsson, H., Astrom, K. J., and Lischinsky, P. (1995). A new model for control of systems with friction. be made quite small. Compared to that, tangential oscillation requires a powerful oscillator (except in the static case), while transverse oscillation is even more energetically expensive, and also less effective overall. There are cases, however, where energy expenditure is not a primary concern (e.g., stabilization of system dynamics) and normal oscillations cannot be easily applied due to technological constraints. In such cases, tangential and even transverse oscillations are viable alternatives.

\section{CONCLUSION}

The present paper summarizes and generalizes a series of recent works that aim to establish a simple macroscopic contact model as a viable explanation for active control of friction by externally applied vibration. Despite its apparent simplicity, the model not only captures the full range of experimentally observed effects, but is also very flexible, being able to adapt to static and dynamic friction, oscillations in normal, tangential and transverse directions, contacts of curved bodies, etc. Apart from straight-forward reduction-of-friction settings, the model can also be applied to the study of frictional drives and actuators under complicated loading scenarios. A similar approach was also highly successful in modeling positioning systems without using any modified friction laws such as the elastoplastic model (see e.g., Teidelt et al., 2012; Grzemba et al., 2014; Teidelt, 2015).

\section{DATA AVAILABILITY STATEMENT}

All datasets generated for this study are included in the article/supplementary material.

\section{AUTHOR CONTRIBUTIONS}

MP conceived and conducted the research and prepared the manuscript.

\section{ACKNOWLEDGMENTS}

This work was supported in part by the Tomsk State University competitiveness improvement programme, which the author gratefully acknowledges.
IEEE Trans. Autom. Control 40, 419-425. doi: 10.1109/9.37 6053

Eaves, A., Smith, A., Waterhouse, W., and Sansome, D. (1975). Review of the application of ultrasonic vibrations to deforming metals. Ultrasonics $13,162-$ 170. doi: 10.1016/0041-624X(75)90085-2

Eggers, G., Klein, J., Blank, J., and Hassfeld, S. (2004). Piezosurgery®: an ultrasound device for cutting bone and its use and limitations in maxillofacial surgery. Br. J. Oral Maxillof. Surg. 42, 451-453. doi: 10.1016/j.bjoms.2004.04.006

Fridman, H. D., and Levesque, P. (1959). Reduction of static friction by sonic vibrations. J. Appl. Phys. 30, 1572-1575. doi: 10.1063/1.1 735002

Gaberson, H. A. (1971). Particle motion on oscillating conveyors: the equations of motion and the rules for predicting motion form transitions. J. Eng. Ind.. 94, 50-56. doi: 10.1115/1.3428154 
Gaberson, H. A. (1972). Particle motion on oscillating conveyors-part 2. practical solutions to the equations of motion and the extension of the theory to beds of granular material. J. Eng. Ind. 94, 57-63. doi: 10.1115/1.3428155

Godfrey, D. (1967). Vibration reduces metal to metal contact and causes an apparent reduction in friction. ASLE Trans. 10, 183-192. doi: 10.1080/05698196708972178

Grzemba, B., Pohrt, R., Teidelt, E., and Popov, V. L. (2014). Maximum microslip in tangential contact of randomly rough self-affine surfaces. Wear 309, 256-258. doi: 10.1016/j.wear.2013.11.050

Heckl, M. A., and Huang, X. (2000). Curve squeal of train wheels, part 3: active control. J. Sound Vibrat. 229, 709-735. doi: 10.1006/jsvi.1999.2512

Mao, X., Popov, V. L., Starcevic, J., and Popov, M. (2017). Reduction of friction by normal oscillations. ii. in-plane system dynamics. Friction 5, 194-206. doi: 10.1007/s40544-017-0146-x

Müller, M., and Ostermeyer, G. (2007). Cellular automata method for macroscopic surface and friction dynamics in brake systems. Tribol. Int. 40, 942-952. doi: 10.1016/j.triboint.2006.02.045

Murakawa, M., and Jin, M. (2001). The utility of radially and ultrasonically vibrated dies in the wire drawing process. J. Mater. Process. Technol. 113, 81-86. doi: 10.1016/S0924-0136(01)00635-5

Pohlman, R., and Lehfeldt, E. (1966). Influence of ultrasonic vibration on metallic friction. Ultrasonics 4, 178-185. doi: 10.1016/0041-624X(66)90244-7

Popov, M., and Li, Q. (2018). Multimode active control of friction, dynamic ratchets and actuators. Phys. Mesomech. 21, 24-31. doi: 10.1134/S1029959918010046

Popov, M., Popov, V. L., and Popov, N. V. (2017). Reduction of friction by normal oscillations. I. influence of contact stiffness. Friction 5, 45-55. doi: 10.1007/s40544-016-0136-4

Popov, V. L. (2017). Oscillation-based methods for actuation and manipulation of nano-objects. AIP Conf. Proc. 1882:020056. doi: 10.1063/1.5001635

Popov, V. L., and Heß, M. (2016). Method of Dimensionality Reduction in Contact Mechanics and Friction. Berlin: Springer. doi: 10.1007/978-3-642-53876-6

Popov, V. L., Starcevic, J., and Filippov, A. (2010). Influence of ultrasonic inplane oscillations on static and sliding friction and intrinsic length scale of dry friction processes. Tribol. Lett. 39, 25-30. doi: 10.1007/s11249-009-9 531-6

Schmidt, J. P., Hagedorn, P., and Bingqi, M. (1996). A note on the contact problem in an ultrasonic travelling wave motor. Int. J. Nonlin. Mech. 31, 915-924. doi: 10.1016/S0020-7462(96)00112-6

Siegert, K., and Ulmer, J. (2001). Superimposing ultrasonic waves on the dies in tube and wire drawing. J. Eng. Mater. Technol. 123, 517-523. doi: 10.1115/1.1397779

Socoliuc, A., Gnecco, E., Maier, S., Pfeiffer, O., Baratoff, A., Bennewitz, R., and Meyer, E. (2006). Atomic-scale control of friction by actuation of nanometersized contacts. Science 313, 207-210. doi: 10.1126/science.1125874

Storck, H., Littmann, W., Wallaschek, J., and Mracek, M. (2002). The effect of friction reduction in presence of ultrasonic vibrations and its relevance to travelling wave ultrasonic motors. Ultrasonics 40, 379-383. doi: 10.1016/S0041-624X(02)00126-9

Teidelt, E. (2015). Oscillating contacts: friction induced motion and control of friction (Ph.D. thesis). TU Berlin, Berlin, Germany.

Teidelt, E., Willert, E., Filippov, A., and Popov, V. L. (2012). Modeling of the dynamic contact in stick-slip microdrives using the method of reduction of dimensionality. Phys. Mesomech. 15, 287-292. doi: 10.1134/S1029959912030071

Thoe, T., Aspinwall, D., and Wise, M. (1998). Review on ultrasonic machining. Int. J. Mach. Tools Manuf. 38, 239-255. doi: 10.1016/S0890-6955(97)00036-9

Conflict of Interest: The author declares that the research was conducted in the absence of any commercial or financial relationships that could be construed as a potential conflict of interest.

Copyright (C) 2020 Popov. This is an open-access article distributed under the terms of the Creative Commons Attribution License (CC BY). The use, distribution or reproduction in other forums is permitted, provided the original author(s) and the copyright owner(s) are credited and that the original publication in this journal is cited, in accordance with accepted academic practice. No use, distribution or reproduction is permitted which does not comply with these terms. 